\title{
Room-temperature coherent revival in an ensemble of quantum dots
}

\author{
Igor Khanonkin $\odot,{ }^{1, *}$ Ori Eyal $\odot,{ }^{1}$ Johann Peter Reithmaier $\odot,{ }^{2}$ and Gadi Eisenstein ${ }^{1}$ \\ ${ }^{1}$ Andrew and Erna Viterbi Department of Electrical Engineering and Russell Berrie Nanotechnology Institute, Technion, Haifa 32000, Israel \\ ${ }^{2}$ Institute of Nanostructure Technologies and Analytics, Technische Physik, CINSaT, University of Kassel, Kassel 34132, Germany
}

(Received 1 November 2020; revised 18 June 2021; accepted 29 June 2021; published 20 July 2021)

\begin{abstract}
We demonstrate the hallmark concept of periodic collapse and revival of coherence in a room-temperature ensemble of quantum dots (QDs) in the form of a 1.5-mm-long optical amplifier. Femtosecond excitation pulses induce coherent interactions with a number of discrete homogeneous QD subgroups within an inhomogeneously broadened ensemble, which interfere constructively to induce coherent revival (CR). The amplitude decay of $\mathrm{CR}$ is dictated by the QD homogeneous linewidth, thus enabling its extraction in a double-pulse Ramsey-type experiment. The more common photon echo technique is also invoked and yields the same linewidth provided that both experiments use the same bias. A dephasing time $T_{2}$ longer than $5 \mathrm{ps}$ is extracted. This is a record long $T_{2}$ for a room-temperature QD ensemble and testifies to the high quality of the InAs/InP QDs used in the experiments. Measured electrical bias and temperature dependencies of the transverse relaxation times $\left(T_{2}\right.$ and $T_{2}^{*}$ ) enable the determination of the two main decoherence mechanisms: carrier-carrier and carrier-phonon scatterings.
\end{abstract}

DOI: 10.1103/PhysRevResearch.3.033073

\section{INTRODUCTION}

Collapse and revival of wave functions lasting well beyond the classical Cummings decay [1] are hallmark quantum optics phenomena. Originally predicted by Eberly et al. [2] for interaction of a quantized coherent field with a single atom, they were termed quantum coherent revival (QCR). QCR was first demonstrated experimentally by Rempe et al. in 1987 in a one-atom maser [3] and then served as a measure of field quantization in cavity quantum electrodynamics (QED) starting in 1996 [4].

Collapse and revival of wave functions are essentially time domain manifestations of a beating mechanism. In the original QCR, summation over a large average number of photons leads to a beating-like pattern, for instance, of the expectation values of population inversion.

For a coherent interaction with classical fields, similar revivals have been observed. These are termed coherent revivals (CRs). A coherent field can actually be viewed as comprising a number of quantized photon states and, according to [5], approaching a classical field as the number of quanta increases. A spectrally broad excitation may interact coherently with several discrete energy states of the atomic medium and these beat to form a CR. CR was demonstrated with classical excitation fields in trapped atoms [6,7], vibration states of

\footnotetext{
*ikhanonkin@technion.ac.il

Published by the American Physical Society under the terms of the Creative Commons Attribution 4.0 International license. Further distribution of this work must maintain attribution to the author(s) and the published article's title, journal citation, and DOI.
}

gases [8,9], excitons in GaAs quantum wells [10], optical lattices [11], and heavy-Fermi-liquid compounds [12].

Thus far, revivals in solids have always required cryogenic temperatures, with no exception. In fact, room temperature is, to date, unequivocally believed to diminish the effect altogether.

Here we report on the demonstration of $\mathrm{CR}$ in a roomtemperature ensemble of semiconductor quantum dots (QDs) operating in the important wavelength range of $1550 \mathrm{~nm}$. The QD ensemble acts as an effective two-level system [13] where the charge carrier band-to-band ground-state transition makes up the two states of a quantum bit (qubit) which are prepared, manipulated, and measured by coherent optical excitation. The platform we used for the CR experiment is a 1.5-mm-long InAs/InP QD optical amplifier operating at room temperature and driven electrically to the gain regime in the 1550-nm wavelength range. We employed the Ramsey configuration with two time-delayed 90 -fs pulses. The excitation pulse induces coherent interactions with a number of discrete groups of QDs (modes) which are a subset of the inhomogeneously distributed QDs. These modes constitute an effective inhomogeneous linewidth that determines an effective inhomogeneous transverse relaxation time $T_{2}^{*}$. This set of modes plays the role of the quantized photon numbers as in Ref. [2] and of elements in a discrete classical spectrum [14], namely, the modes interfere constructively to induce CR. CR can also be viewed in the time domain. The different modes have slightly different transition frequencies and hence accumulate different phases as they precess. At specific times, their phases align and then a revival event takes place.

The decay of the consecutive revival amplitudes is governed by the homogeneous transverse relaxation time $T_{2}$ whose extracted value was confirmed by a three-pulse 


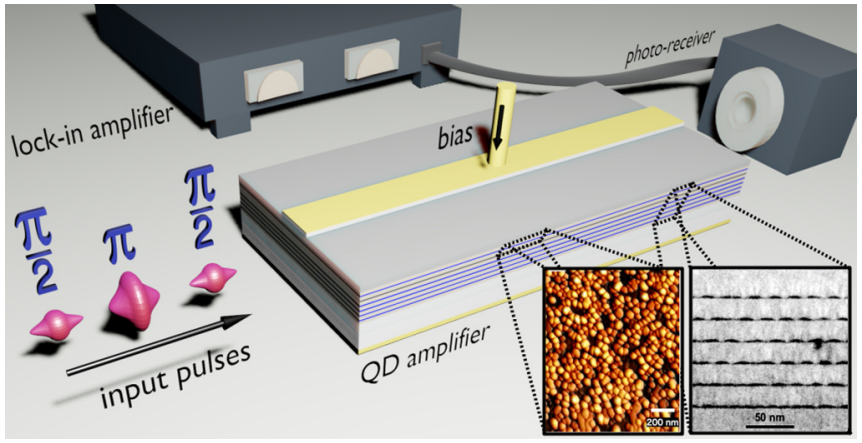

FIG. 1. General description of the experiment showing schematically the $\pi / 2$ area Ramsey pulses and the $\pi$ area rephasing pulse at the input to the QD amplifier. The figure includes an atomic force microscope image of a single layer of self-assembled QDs and a high-resolution transmission electron microscope cross section of the six-QD-layer structure.

photon echo experiment similar to the conventional spin echo experiment $[15,16]$. Finally, we present the measured dependencies of the extracted $T_{2}$ and $T_{2}^{*}$ values on the carrier density [17] and the temperature [18], from which we determine the decoherence mechanisms.

Our findings pave the way to practical elements for quantum information processing, communication, and simulations where coherent states are controlled using compact semiconductor nanostructures operating at room temperature. Moreover, operation at $1550 \mathrm{~nm}$ is compatible with, and opens the way for, direct integration with compact silicon photonics circuits as well as fiber-optic networks.

The advantages of semiconductor nanostructures for quantum devices have long been recognized. Quantum devices and systems based on QDs [19-22], some used in conjunction with photonic crystal waveguides and cavities [23-25], have been studied extensively, as have special nanowires [26-29], which are also thought to be improved by combining them with QDs [30]. Unlike our room-temperature CR demonstration, all these quantum elements operate exclusively at cryogenic temperatures.

\section{EXPERIMENTAL CONDITIONS}

The general experimental configuration we employed is depicted schematically in Fig. 1 and detailed in Appendix A. Ninety-femtosecond pulses were split into two or three for the Ramsey and the photon echo experiments, respectively. The Ramsey pair and the photon echo-rephasing pulses were cross-polarized to TE and TM, respectively. The average power of the second Ramsey pulse was measured using a photoreceiver and a lock-in amplifier at the QD waveguide output. To ensure complete pulse separation at the input, the minimum nominal temporal delay between copolarized pulses was $600 \mathrm{fs}$.

The active region of the gain medium we used comprised six layers of high-density, $6 \times 10^{10} \mathrm{~cm}^{-2}$ InAs QDs grown by molecular beam epitaxy in the Stranski-Krastanow mode. The amplifier was fabricated as a $2-\mu \mathrm{m}$-wide, 1.5 -mm-long ridge waveguide whose end facets were antireflection coated. Figure 1 includes an atomic force microscope image of a single
QD layer as well as a high-resolution transmission electron microscope cross section of the six-layer structure. The QDs exhibit record uniformity characterized by their photoluminescence linewidth at $10 \mathrm{~K}$, which was 17 and $26 \mathrm{meV}$ for a single QD layer and for the six-layer stack, respectively [31]. The emission power of the measured electroluminescence spectra increases with applied bias but the spectral shape remains unchanged [32]. This ensures that for all bias levels, the excitation pulse interacts with the same QDs and the pulse area changes linearly with bias.

During the delay between the Ramsey pair of pulses, the QD state vectors, in a Bloch sphere representation, evolve in a periodic manner (in the rotating frame approximation) at the classical frequency of the atom transition on which an exponential decay, representing damping, is superimposed. The second Ramsey pulse directly probes these damped Ramsey interference fringes and consequently yields the inhomogeneous transverse relaxation time $T_{2}^{*}$. The three-pulse photon echo technique employs an additional excitation pulse (called the rephasing pulse) which is launched in the middle of the Ramsey pair. The rephasing pulse reverses the phases accumulated across the inhomogeneously broadened ensemble so that the system coherence is determined solely by the homogeneous linewidth [33].

The Ramsey method requires that the excitation pulses have areas of $\pi / 2$. In distributed systems, when a propagating pulse experiences gain or absorption, the amplitude and phase profiles change continuously. Therefore, only an effective pulse area can be defined. This is the case in the QD amplifier [34] as well as in other systems, for example, a dense atomic medium. The $\pi / 2$ effective area of the TE polarized Ramsey pulses was set by optimizing their input energy to obtain the largest possible Ramsey interference contrast at a given bias. This ensures that the effective pulse area is as close as possible to $\pi / 2$. In the present experiment, the first and second input pulse energies that yield the closest area to $\pi / 2$, when the amplifier is driven at $7.15 \mathrm{kA} / \mathrm{cm}^{2}$, are $7.5 \mathrm{pJ}$ and $5.6 \mathrm{pJ}$, respectively. The intensity of the $\pi$ area rephasing pulse should be $\sqrt{2}$ larger than that of the $\pi / 2$ Ramsey pulses. However, due to the polarization-dependent dipole moment [determined from gain $(G)$ measurements], the intensity is larger. It was set to be $\sqrt{2} \cdot G_{T E} / G_{T M}$ larger than that of the first Ramsey pulse.

\section{EXPERIMENTAL AND THEORETICAL RESULTS}

The measured intensity of the second Ramsey pulse at the amplifier output for a current density of $7.15 \mathrm{kA} / \mathrm{cm}^{2}$ is presented in the upper part of Fig. 2 for a time span ranging from 600 fs to 15 ps. The initial Ramsey contrast decay, which is governed by $T_{2}^{*}=1.22 \mathrm{ps}$, resembles the Cummings collapse. At later times, four periodic cycles of coherence, representing CRs that originate from the interference of a few excited discrete modes, each with a slightly different transition frequency, are clearly observed. The insets show imprints of Ramsey interference fringes with a periodicity of $\approx 5.1 \mathrm{fs}$, corresponding to one optical cycle at the QD gain peak, $1535 \mathrm{~nm}$. In between the revivals, the response is just noise. 


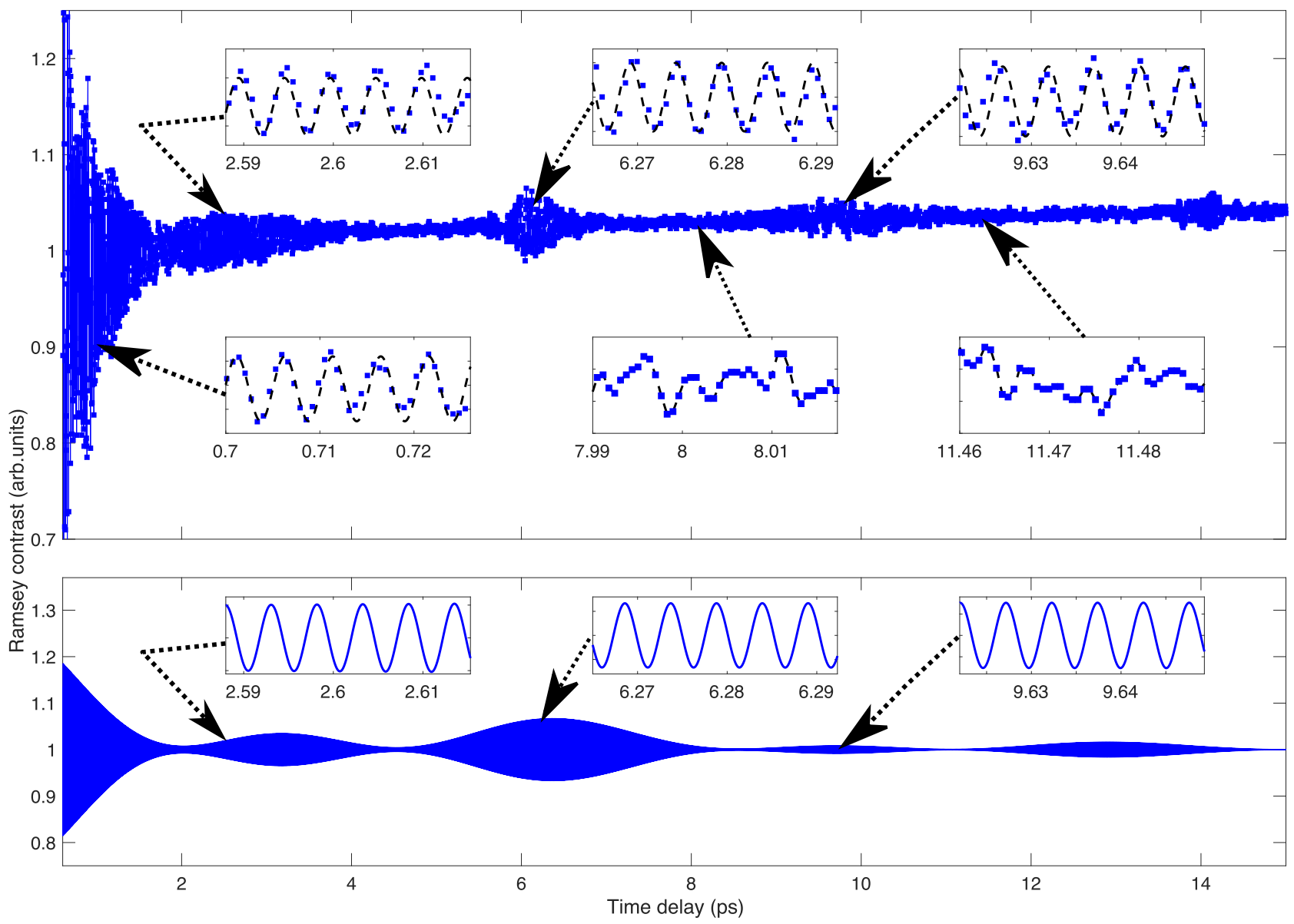

FIG. 2. Top: Measured normalized Ramsey contrast at a current density of $7.15 \mathrm{kA} / \mathrm{cm}^{2}$ revealing CR. Insets: Ramsey fringes with a periodicity of $\approx 5.1 \mathrm{fs}$ corresponding to the QD transition energy at $1535 \mathrm{~nm}$ and noise in between the revivals, shown for clarity. The initial decay is dictated by the effective inhomogeneous transverse relaxation time, $T_{2}^{*}$, while the CR decays according to the homogeneous transverse relaxation time, $T_{2}$. Bottom: Normalized analytical solution of five excited uncoupled modes each with a dephasing time of $T_{2}=4.64 \mathrm{ps}$, spectrally located close to $1535 \mathrm{~nm}$. Inset: Calculated Ramsey fringes, shown for clarity.

The spectral placement of the pulses relative to the $\mathrm{QD}$ gain peak as well as the pulse amplitude and spectral irregularities determine the set of modes to be excited in a coherent manner that can be sensed in a two-pulse Ramsey-type experiment. The particular modes participating in the interaction, for the experimental conditions leading to Fig. 2, were determined using a comprehensive model of a propagating pulse in a QD amplifier [35]. The model, which is detailed in Appendix B, solves the Maxwell and Schrödinger equations in the presence of all nonlinear and nonresonant interactions $[34,36]$. The use of this model avoids the need to solve the Jaynes-Cummings model since the coherent part of the interaction is calculated by the Schrödinger equation while the pulse propagation naturally uses the Maxwell equation.

The simulation enables us to map the population inversion $\left(\rho_{11}-\rho_{22}\right)$ and the coherences $\left(\rho_{12}\right)$ anywhere along the amplifier and at all wavelengths, with $\rho_{i j}$ being elements of the density matrix. Figure 3 describes normalized population inversion and coherences of the QD ensemble when two Ramsey pulses, separated from each other by $1 \mathrm{ps}$, have propagated a few hundred micrometers along the amplifier. The oscillatory nature shows clearly five dominant modes. The spectral signature of the five modes changes somewhat as the pulses propagate along the amplifier but the general shape is maintained.

The measured revival pattern can be reconstructed with those five interacting modes as shown in the lower part

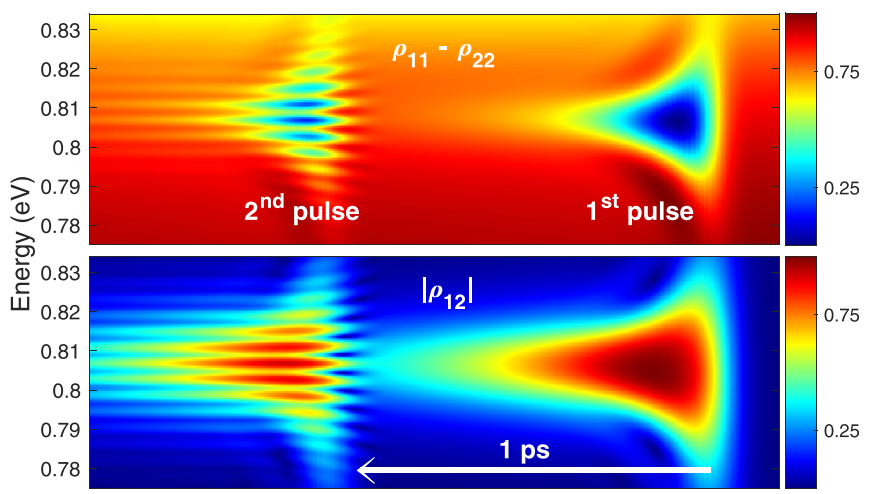

FIG. 3. Simulated Ramsey-type experiment. The spectral dependence of the normalized QD population inversion (upper trace) and coherences (lower trace) upon Ramsey pulse pair propagation with a 1-ps mutual delay for a few hundred micrometers along the amplifier. 

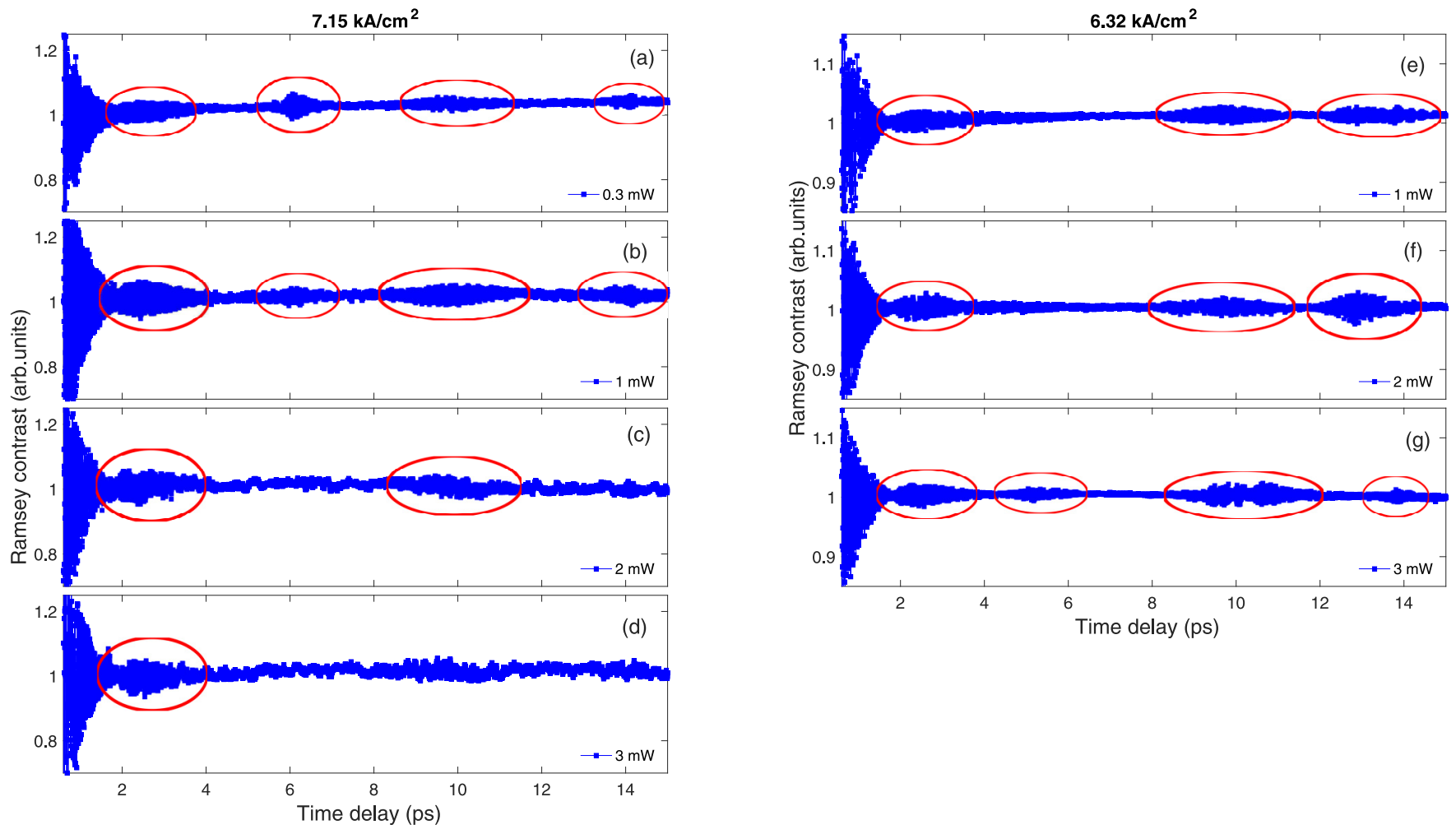

FIG. 4. Measured normalized Ramsey contrast at current densities of $7.15 \mathrm{kA} / \mathrm{cm}^{2}$ (left column) and $6.32 \mathrm{kA} / \mathrm{cm}^{2}$ (right column) revealing the CR pattern variations with an increase in the excitation Ramsey pulses intensities.

of Fig. 2. An analytical time-resolved response is used, $\exp \left(-t / T_{2}\right) \cdot \sum_{k} a_{k} \sin \left(2 \pi t / t_{k}\right)$, where the five interacting modes are considered to be uncoupled with weighted amplitudes $a_{k}=\{1 / 3,1 / 2,1,1 / 2,1 / 3\}$ and oscillation periods $t_{k}=(5.109+k \cdot 0.004)$ fs. Each mode is broadened to $1.79 \mathrm{meV}$, leading to a dephasing time $T_{2}=$ 4.64 ps. The analytical calculation fits the experimental result very well.

To further investigate the details of our CR observation, we measured the revival patterns for different excitation powers and for an additional bias. Figure 4 depicts the CR patterns for Ramsey excitation pulse powers ranging from 0.3 to $4 \mathrm{~mW}$ when the amplifier is biased at two levels, 7.15 and $6.32 \mathrm{kA} / \mathrm{cm}^{2}$. For the higher bias, the trace in Fig. 4(a) is a replica of the pattern in Fig. 2. A pulse power increase from 0.3 to $1 \mathrm{~mW}$ [trace in Fig. 4(b)] inverts the revival amplitude sequence to become large-small-large-small. A further increase in the pulse intensities to $2 \mathrm{~mW}$ [trace in Fig. 4(c)] leads to a pattern with only two revivals (first and third). For 3-mW pulses [trace in Fig. 4(d)], only the first revival is clearly seen; the third revival almost disappears and the second and fourth are totally absent. The experiment was repeated at a lower bias level: $6.32 \mathrm{kA} / \mathrm{cm}^{2}$. Figures $4(\mathrm{e})-4(\mathrm{~g})$ show the revival patterns for three input powers. At $1 \mathrm{~mW}$ [trace in Fig. 4(e)], only three revivals are seen. At $2 \mathrm{~mW}$ [trace in Fig. 4(f)], there are also three revivals, with the third being larger than that in Fig. 4(e). At the low bias, the difference between the revivals in this input power regime is small. However, at $4 \mathrm{~mW}$ [trace in Fig. 4(g)] a clear fourth revival appears. The last revival (near $14 \mathrm{ps)} \mathrm{is} \mathrm{reduced} \mathrm{in} \mathrm{amplitude} \mathrm{but} \mathrm{a} \mathrm{new} \mathrm{revival} \mathrm{appears}$ at roughly 5 ps.
In all Ramsey experiments, the interference frequency is determined by the transition frequency of the atom [37]. In the present case, the QD ensemble acts as a group of effective two-level systems which have ground-state transition frequencies that differ in a very minor manner. Therefore, they appear in the measurement as an average frequency near that of the gain peak. An exemplary Fourier transform of the CR response shown in Fig. 4(b) is presented in Fig. 5. The Fourier

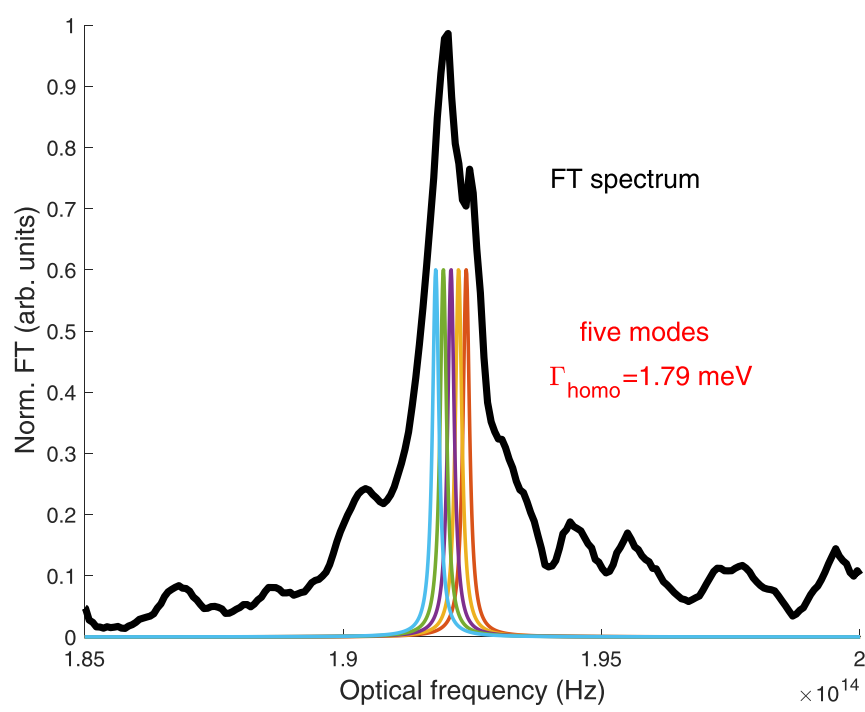

FIG. 5. Fourier transform of the measured Ramsey contrast at a current density of $7.15 \mathrm{kA} / \mathrm{cm}^{2}$ revealing the QD mode spectral extent that induces the observed $\mathrm{CR}$ pattern. 


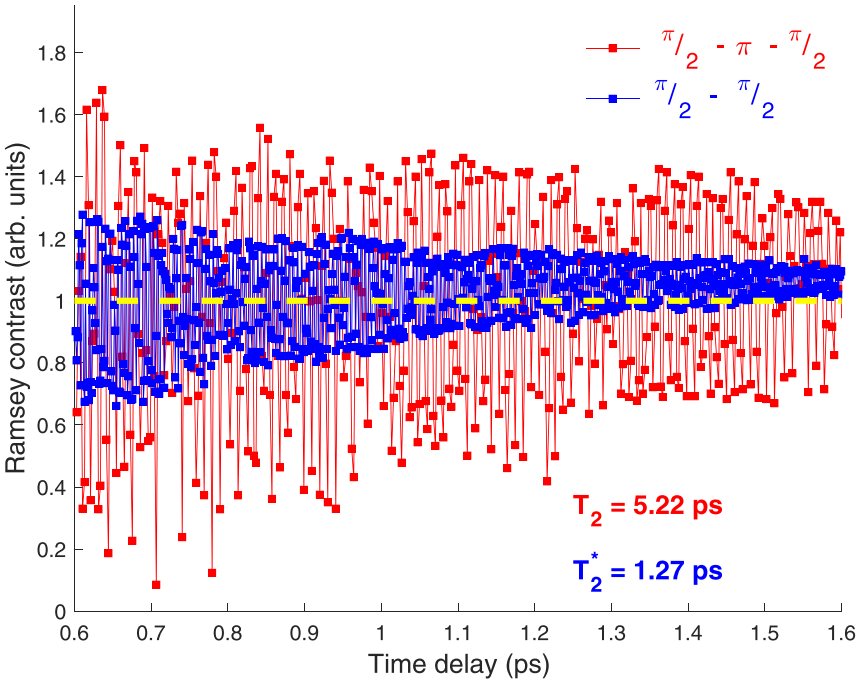

FIG. 6. Contrast of Ramsey interference fringes measured at a current density of $4.7 \mathrm{kA} / \mathrm{cm}^{2}$ in two-pulse (blue trace) and threepulse (red trace) experiments. The coherence decays according to $T_{2}^{*}=1.27 \mathrm{ps}$ extracted from the blue trace. The three-pulse photon echo experiment yields $T_{2}=5.22 \mathrm{ps}$. The yellow line of the contrast equal to 1 is shown as a guide for the eye.

transform spectral width contains the five QD modes used to reproduce the CR pattern depicted at the bottom of Fig. 2.

The initial exponential decay in Fig. 2 provides the effective inhomogeneous transverse relaxation time $T_{2}^{*}$. The individual modes are, by definition, homogeneous and their coherence is characterized by the dephasing time $T_{2}$. The interaction between modes induces the $\mathrm{CR}$ and therefore the $\mathrm{CR}$ decay is governed by $T_{2}$. To confirm the latter, we performed a three-pulse photon echo measurement. Figure 6 compares the Ramsey contrast, measured with the standard Ramsey technique (blue trace), with that measured in the induced photon echo configuration (red trace) for a current density of $4.7 \mathrm{kA} / \mathrm{cm}^{2}$. Clearly, the photon echo technique prolongs the QD coherence substantially. Using the two experiments we fit the measured Ramsey contrasts according to $\exp \left(-2 t / T_{2}^{*}\right)$ and $\exp \left(-4 t / T_{2}\right)$ [38] and extract the two time constants, $T_{2}^{*}=1.27 \mathrm{ps}$ and $T_{2}=5.22 \mathrm{ps}$, respectively. For a larger bias, the dephasing time shortens and at $7.15 \mathrm{kA} / \mathrm{cm}^{2}$, which is the bias used in Fig. 2, it decreases to 4.64 ps, matching the $T_{2}$ value extracted from the CR decay. The extracted $T_{2}$ values are significantly higher than those published previously for QDs in the GaAs material system $[18,39]$. The measured long dephasing times testify to the high quality of the high-density InAs/InP QDs, which are extremely uniform [31]. Indeed, such QDs act as an atomlike gain material [40] which enables lasers with record performance in terms of modal gain, temperature independence [41], and linewidth [42].

The experimental traces in Fig. 6 are not symmetric relative to the time axis. This asymmetry stems from nonlinear absorption of the first Ramsey pulse. Charge carriers absorbed during the incoherent nonlinear interaction relax to the QD ground states on a picosecond time scale and provide additional gain Ref. [32]. This problem was overcome by averaging the upper and lower exponential decays of the fringe envelopes.

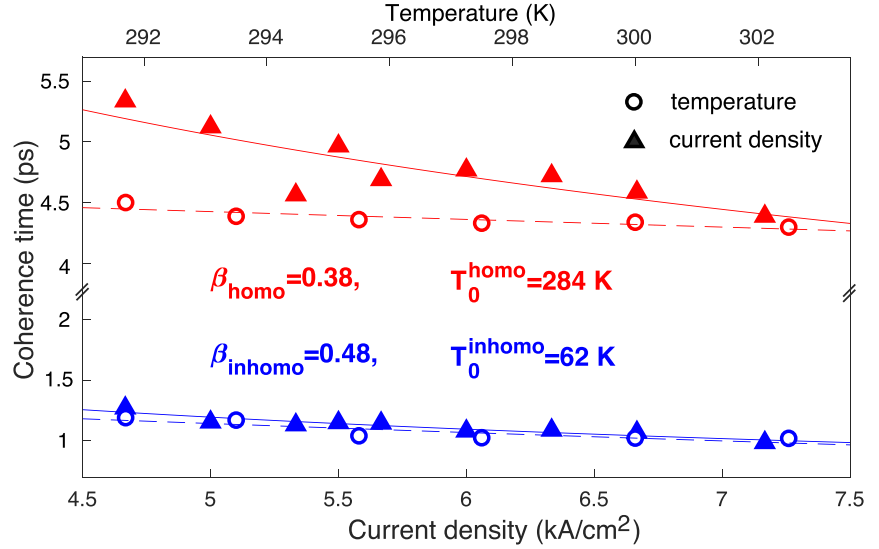

FIG. 7. $T_{2}$ (red trace) and $T_{2}^{*}$ (blue trace) dependencies on the current density and temperature representing carrier-carrier and carrier-phonon scatterings. The current density dependencies follow a power law $\propto n^{-\beta}$ yielding the homogeneous broadened $\beta_{\text {homo }}=$ 0.38 and inhomogeneous broadened $\beta_{\text {inhomo }}=0.48$. The temperature dependencies are fitted with an exponential function $\propto \exp \left(-T / T_{0}\right)$ yielding $T_{0}^{\text {homo }}=284 \mathrm{~K}$ and $T_{0}^{\text {inhomo }}=62 \mathrm{~K}$.

As the Ramsey coherent interference has a time scale of a few femtoseconds, the contrast is unaffected by the incoherent nonlinear propagation.

Operation at room temperature affects the system decoherence significantly and therefore it is crucial to understand, in detail, the mechanisms causing decoherence. There are two main mechanisms of decoherence [43]: carrier-carrier scattering, originating from Coulomb interaction between QD confined charge carriers and the surrounding area; and carrierphonon scattering, involving acoustic and longitudinal optical phonons. These two mechanisms were examined by measuring $T_{2}^{*}$ and $T_{2}$ in double- and triple-pulse experiments for various applied carrier densities ( $n$ ) and temperatures $(T)$. Figure 7 summarizes the extracted characteristic times with a predicted scaling of $\propto n^{-\beta}$ and $\alpha \exp \left(-T / T_{0}\right)$ for the bias and temperature dependencies, respectively. $T_{2}^{*}$ was found to be less dependent on the carrier density compared with $T_{2}$. The extracted fitting coefficients are $\beta_{\text {homo }}=0.38$ and $\beta_{\text {inhomo }}=0.48$. For carrier-carrier scattering, these coefficients are known for GaAs in the bulk $\left(\beta_{\text {homo }}^{\text {bulk }}=0.3\right)[44]$ and in GaAs quantum wells $\left(\beta_{\text {homo }}^{\text {well }}=0.5\right)$ [45]. A theory [17] predicts $\beta_{\mathrm{homo}}^{\mathrm{QD}}=0.56$ in shallow $\mathrm{QDs}$ and $\beta_{\mathrm{homo}}^{\mathrm{QD}}=0.63$ in deep QDs. An exact comparison with the obtained value for the QDs is difficult due to the different dimensionality and material compositions as well as the fact that in the QD structure, some of the carrier-carrier scatterings take place in the quantum well-like high-energy barrier. Nevertheless, the obtained value of $\beta_{\text {homo }}$ is in the right range and sheds light on the origin of the scattering events. The value of $\beta_{\text {inhomo }}$ cannot be compared to that in a quantum well or in the bulk since those are homogeneous systems.

Figure 7 describes also an almost-constant $T_{2}$ in the relatively narrow, measured temperature range, while $T_{2}^{*}$ appears to be strongly dependent on the temperature; the extracted fitting coefficients are $T_{0}^{\text {homo }}=284 \mathrm{~K}$ and $T_{0}^{\text {inhomo }}=62 \mathrm{~K}$, respectively. Since at room temperature the separation between the first excited energy state ( $p$ shell) and the ground 
(a) Positive QSP
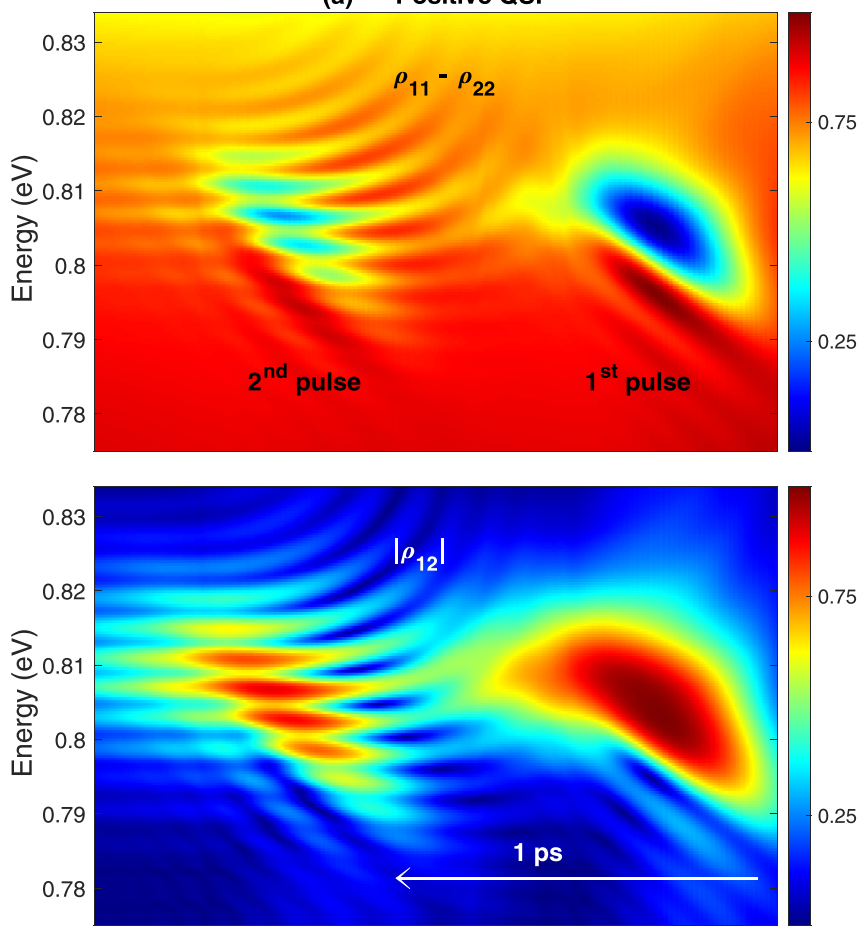

(b) Negative QSP
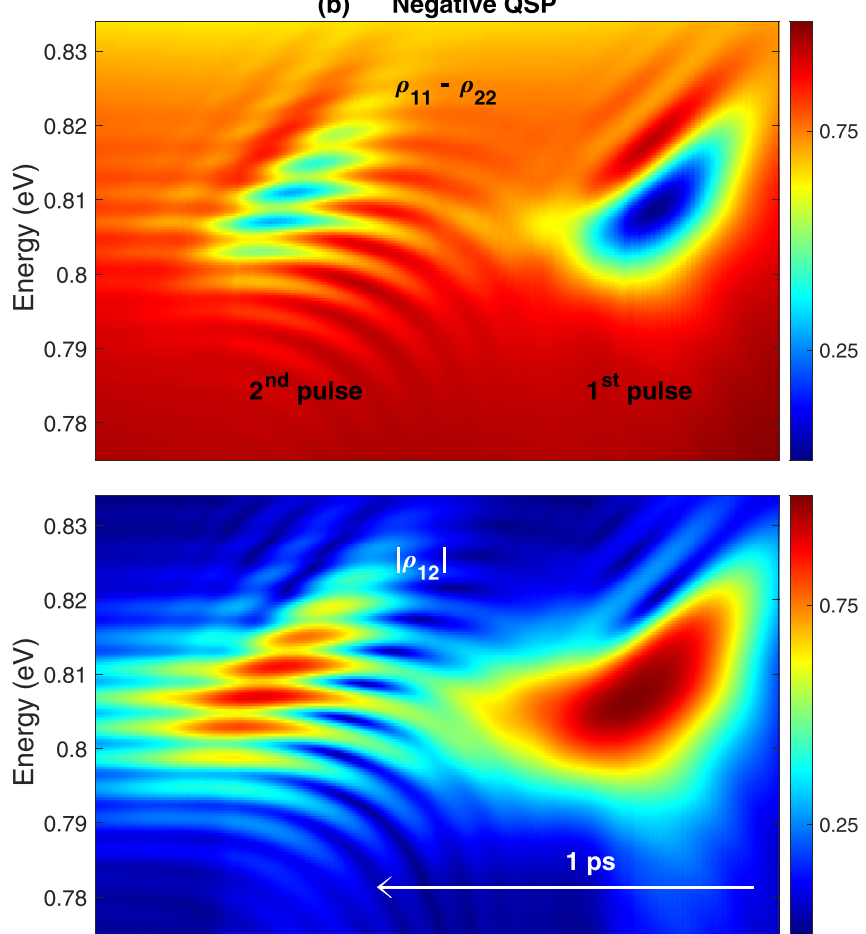

FIG. 8. Simulated Ramsey-type experiment, similar to Fig. 3, for the chirped excitation pulses. The spectral dependence of the normalized QD population inversion (upper trace) and coherences (lower trace) for a Ramsey pulse pair that is initially chirped with (a) a positive and (b) a negative quadratic spectral phase (QSP), 0.25 and $-0.25 \mathrm{ps}^{2}$, respectively.

state ( $\mathrm{s}$ shell) in the InAs QDs is $\approx 60 \mathrm{meV}$ [32], which equals roughly the energy of two longitudinal optical phonons ( $\approx 30 \mathrm{meV}$ [46]), relaxation by carrier-phonon interaction is probable as observed in the case of GaAs QDs with multiple longitudinal optical phonon resonances whose homogeneous broadening was shown to be temperature dependent above $200 \mathrm{~K}$ [18].

\section{DISCUSSION AND CONCLUSION}

We have demonstrated the important phenomenon of CR in a room-temperature semiconductor QD-based optical amplifier driven by an electrical bias to the gain regime in the wavelength range of $1550 \mathrm{~nm}$. CR manifests itself as periodic recurrences of the coherence which originate from constructive interference between excited homogeneous QD subgroups. The coherent interference of the QD modes persists up to the characteristic homogeneous time $T_{2}$, thus enabling its extraction without the need to invoke the photon echo technique.

In the present experiment, the properties of the excitation pulse spectrum determine the number of interacting modes to be five, and this leads to the particular revival sequence shown in Fig. 2. The present configuration prolongs the coherence to 5.22 ps. Longer dephasing times are possible, in principle, if multiple $\pi$ pulses are placed between the two $\pi / 2$ pulses [47].

Semiconductor QDs are the most attractive solid-state platform for quantum information processing, communication, and simulation. QD amplifiers, similar to those employed here, have actually been shown to maintain their properties at temperatures as high as $100^{\circ} \mathrm{C}$ when used in telecom applications [48]. We envision therefore a host of compact future quantum devices which do not require cryogenic cooling. For example, shaping the excitation pulses using a spatial light modulator will allow careful control over the modes that participate in the coherent interaction, thereby determining specific $\mathrm{CR}$ patterns which can serve as quantum codes. In principle, each mode (consisting of a macroscopic quantity of QDs) can be controlled separately by shaped excitation pulses transforming the QD ensemble into several independent qubits within a single device which are addressed simultaneously. This is confirmed by the simulation results shown in Fig. 8. The initially chirped excitation pulses induce coherent interactions with QDs of different energies, namely, the positive (or negative) quadratic spectral phase pulses excite QDs spectrally placed on the low-energy (or high-energy) side of the gain peak, respectively. Furthermore, in a single-pulse experiment with chirped excitation pulses, which are spectrally detuned relative to the QD gain peak, Rabi oscillations could be enhanced or diminished, as reported in Ref. [49]. Similarly, the excitation pulses with a positive QSP, located on the high-energy side of the QD gain peak [Fig. 9(a)], interact coherently with a larger number of QDs from the gain peak vicinity and, thus, cause an enhanced $\mathrm{CR}$ interaction. CR imprints are almost concealed for the negative QSP pulses, which are located on the high-energy side of the QD gain peak [Fig. 9(b)], due to a low QD number occupying the high-energy side of the spectrum. The opposite occurs for pulses placed on the low-energy side of the QD 

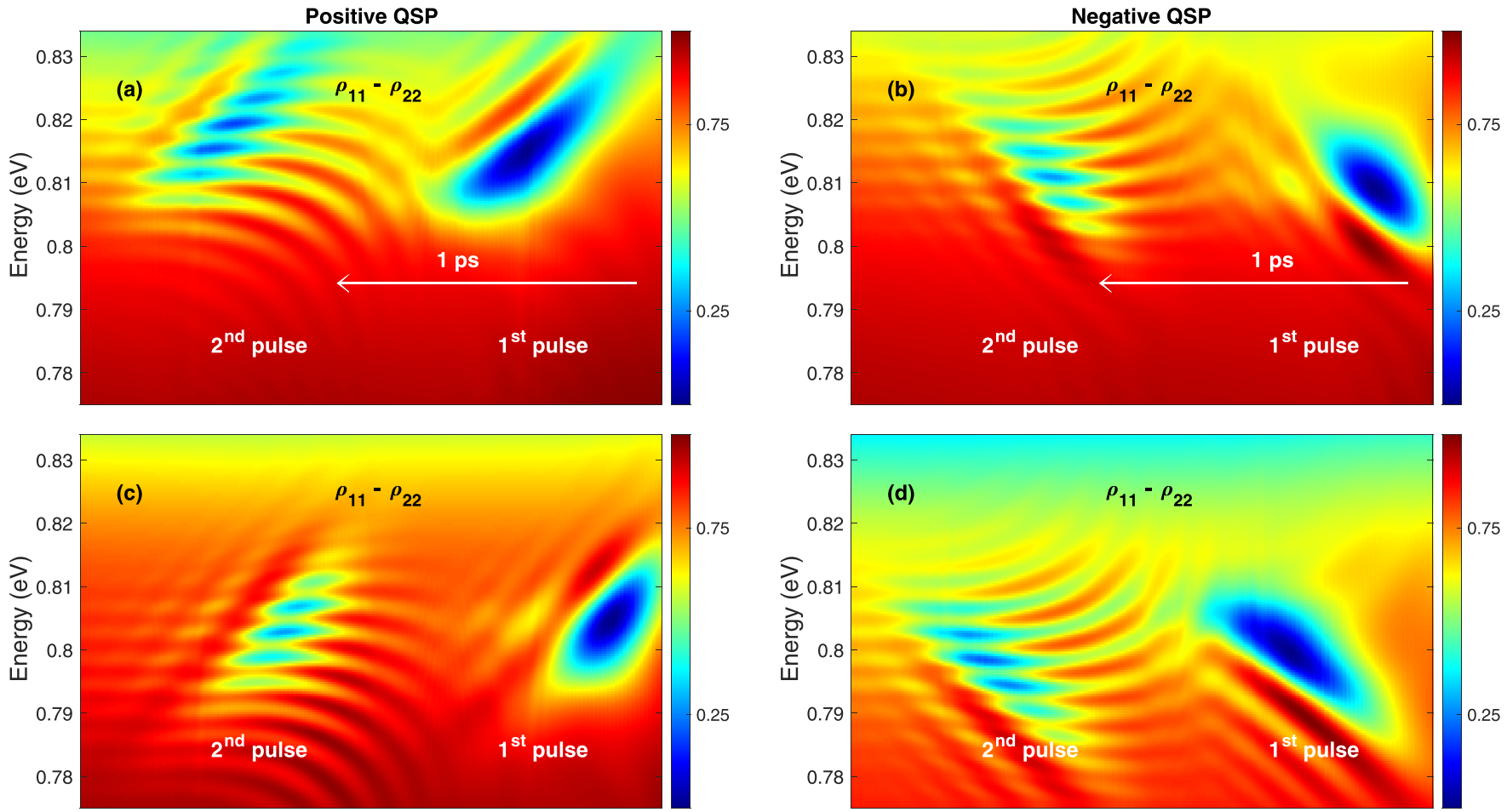

FIG. 9. Spectral dependence of the normalized QD population inversion in the simulated Ramsey-type experiment for chirped excitation pulses. The pulses are spectrally located on the high-energy (a), (b) and low-energy (c), (d) side of the QD gain peak. The Ramsey pulse pair is initially chirped with positive (a), (c) and negative (b), (d) quadratic spectral phases (QSPs) with values of 0.25 and $-0.25 \mathrm{ps}^{2}$, respectively.

gain peak [Figs. 9(c) and 9(d)]. A more complicated spectral shaping of excitation pulses will enable a flexible and versatile control over the excited QD modes.

The room-temperature coherent imprints in the QD ensemble, in the form of CR, occur on a time scale of 15 ps. This will enable the use of standard fast optical detectors with bandwidths of tens of gigahertz, used routinely in telecom technologies. Finally, operation at $1550 \mathrm{~nm}$ is ideal for direct integration with silicon photonic circuits and fiber-optic systems. Obviously, the prospect of operating quantum devices at elevated temperatures advances their practical perspective significantly.

\section{ACKNOWLEDGMENTS}

The authors are grateful to Prof. Moti Segev, Prof. Ariel Kaplan, and Prof. Eric Akkerman for important suggestions during the course of this research. I.K. thanks the Helen Diller Quantum Center and the Jacobs Foundation for financial support. This research was partially funded by the Israel Science Foundation, Grant No. 1504/16.

\section{APPENDIX A}

The detailed experimental configuration is depicted schematically in Fig. 10(a). A TOptica FemtoFiber Pro laser generated nearly transform limited 90 -fs pulses at a repetition rate of $40 \mathrm{MHz}$. Figure 10(b) shows the reconstructed amplitude and instantaneous frequency profiles of the pulses that were characterized by the frequency-resolved optical gating
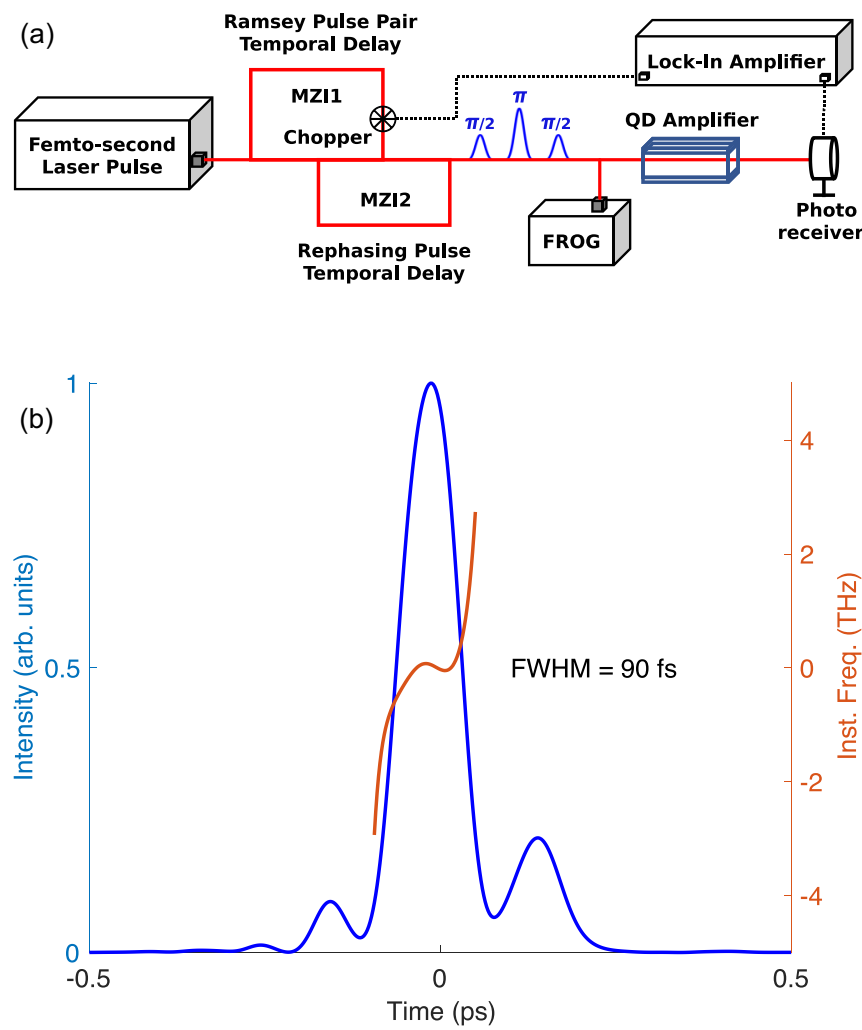

FIG. 10. (a) Detailed schematic of the experimental system. (b) Amplitude and instantaneous frequency profiles of the $90-\mathrm{fs}$ pulses obtained by a FROG measurement. 
(FROG) technique [50]. The pulses were split, respectively, into two or three for the Ramsey and the photon echo experiments using Mach-Zehnder-type interferometers. The delays between pulses were controlled with a subfemtosecond resolution, achieved using accurate DC motorized linear stages, Newport VP-25XA and PI M-126. The Ramsey pair and the rephasing pulses were cross-polarized to TE and TM, respectively. The second Ramsey pulse was chopped, and its average power was measured using a Ge-based photoreceiver and a Stanford SR830 lock-in amplifier at the QD waveguide output. The "time delay" in Figs. 2, 4, and 6 refers to nominal temporal Ramsey pulse pair separations, which were no less than $600 \mathrm{fs}$, to ensure complete pulse separation.

\section{APPENDIX B}

The theoretical investigation of ultrashort pulse propagation in a QD amplifier operating at room temperature is based on a semiclassical description of the light-matter interaction [51], solved in the dipole moment approximation. We employ a numerical finite-difference time-domain model, developed in Refs. [32,34,35], that solves Schrödinger equations for the occupation probabilities of a cascade of two-level quantum systems having different transition energies that represent the inhomogeneously broadened ensemble of QDs. Simultaneously, it solves Maxwell's equation for the electromagnetic field of the propagating pulse, where the vector polarization includes contributions from the interaction with the QDs, from two-photon absorption (TPA) and its accompanying Kerr-like effect, and from group velocity dispersion and the refractive index dependence on the carrier population, known as the plasma effect.

The time evolutions of the QD occupation probabilities in the upper $\left(\rho_{11}^{i}\right)$ and lower $\left(\rho_{22}^{i}\right)$ states, together with the coherences $\left[\rho_{12}^{i}=\left(\rho_{21}^{i}\right)^{*}\right]$, are calculated by the following coupled equations:

$$
\begin{aligned}
& \frac{\partial \rho_{11}^{i}}{\partial t}=-\gamma_{c} \rho_{11}^{i}+\frac{N_{e x}^{i}}{2 N_{D_{\text {total }}} \tau_{e x \rightarrow 11}}\left(1-\rho_{11}^{i}\right) \\
& +\frac{N_{\text {res }}}{2 N_{D_{\text {total }}} \tau_{d_{\text {cap }}}}\left(1-\rho_{11}^{i}\right)-\frac{\rho_{11}^{i}}{\tau_{d_{\text {esc }}^{i}}^{i}}\left(1-\frac{N_{\text {res }}}{D_{\text {res }}}\right) \\
& -\frac{\rho_{11}^{i}}{\tau_{d_{\mathrm{esc}}}^{i}}\left(1-\frac{N_{\mathrm{res}}}{D_{\mathrm{res}}}\right)-j \frac{\vec{\mu} \cdot \vec{E}}{\hbar}\left(\rho_{12}^{i}-\rho_{21}^{i}\right) \text {; } \\
& \frac{\partial \rho_{22}^{i}}{\partial t}=-\gamma_{v} \rho_{22}^{i}-\frac{P_{\text {res }}}{\tau_{\text {cap }}^{h}} \frac{\rho_{22}^{i}}{2 N_{D_{\text {total }}}} \\
& +\frac{\left(1-\rho_{22}^{i}\right)}{\tau_{\mathrm{esc}}^{h}}\left(1-\frac{P_{\mathrm{res}}}{D_{\mathrm{res}}}\right)+j \frac{\vec{\mu} \cdot \vec{E}}{\hbar}\left(\rho_{12}^{i}-\rho_{21}^{i}\right) \text {; } \\
& \frac{\partial \rho_{12}^{i}}{\partial t}=-\left(j \omega+\gamma_{h}\right) \rho_{12}^{i}-j \frac{\vec{\mu} \cdot \vec{E}}{\hbar}\left(\rho_{11}^{i}-\rho_{22}^{i}\right) \text {. }
\end{aligned}
$$

$N_{\text {res }}$ and $P_{\text {res }}$ are, respectively, the electron and hole densities in the corresponding reservoirs and excited states. $N_{e x}^{i}$ is the excited-state carrier density in the $i$ th QD group, with the corresponding recombination time $\tau_{e x}$. The terms $\mu$ and $E$ in the Schrödinger equations are the dipole moment of QDs and the electric field, respectively. $\gamma_{c}$ and $\gamma_{v}$ are the relaxation rates of the upper and the lower levels and $\gamma_{h}$ determines the homogeneous linewidth. $\tau_{\text {cap }}, \tau_{\text {esc }}, \tau_{d_{\text {cap }}}$, and $\tau_{d_{\mathrm{esc}}}$ represent the time constants for capture and escape events for cascaded (cap, esc) and direct $\left(d_{\text {cap }}, d_{\text {esc }}\right)$ transitions, respectively, between the ground QD state, the associated excited state, and the electron reservoir.

The Maxwell curl equations are solved simultaneously for a TE polarized propagating electromagnetic wave,

$$
\frac{\partial E_{x}}{\partial z}=-\frac{\partial B_{y}}{\partial t}, \quad-\frac{\partial H_{y}}{\partial z}=\frac{\partial D_{x}}{\partial t},
$$

where $E_{x}$ and $H_{y}$ are the electric field and magnetic field components, respectively. The electric displacement $D_{x}$ and magnetic field $B_{y}$ read as

$$
D_{x}=\varepsilon_{0} E_{x}+P_{x}, \quad B_{y}=\mu_{0} H_{y} ;
$$

$\varepsilon_{0}$ and $\mu_{0}$ are the vacuum permittivity and permeability. Interaction with the material perturbs the polarization $P_{x}$, which includes several components,

$$
P_{x}=P_{\mathrm{disp}}+P_{\mathrm{QD}}+P_{\mathrm{plasma}}+P_{\mathrm{TPA}}+P_{\mathrm{Kerr}} ;
$$

they are, respectively, dispersion, radiation of the two-level systems, the plasma effect, two-photon absorption, and its accompanying Kerr-like effect.

The linear dispersion contribution to the polarization term [52] is simulated using the Lorentz model neglecting the contribution from damping,

$$
P_{\text {disp }}(\omega)=\varepsilon_{0} \frac{f_{s} \omega_{\text {res }}^{2} E_{x}}{\omega_{\text {res }}^{2}-\omega^{2}},
$$

where $f_{s}$ is the oscillator strength and $\omega_{\text {res }}$ is the resonant frequency of the Lorentz oscillator. Those parameters can be obtained by solving the coupled equations for the chosen values of the dielectric permittivity and the group velocity dispersion.

The induced polarization by the QDs [35] is

$$
P_{\mathrm{QD}}=2 \mu_{x} \cdot \sum_{\text {ensemble }} N_{D}^{i}\left(\rho_{12}^{i}+\rho_{21}^{* i}\right) \Gamma,
$$

where $\Gamma$ is a confinement factor.

The TPA contribution to the polarization can be expressed as $P_{\mathrm{TPA}}=\varepsilon_{0} \chi_{\mathrm{TPA}} E_{x}$, where the $\chi_{\mathrm{TPA}}$ as a function of the TPA coefficient $\beta_{\mathrm{TPA}}$, defined by Beer's law (the optical intensity change due to TPA upon propagation $\left.\frac{d I}{d t}=-\beta_{\mathrm{TPA}} I^{2}\right)$,

$$
\chi_{\mathrm{TPA}}=\frac{c^{2} \varepsilon_{0} n_{0}^{2} \beta_{\mathrm{TPA}}}{2 i \omega}\left|E_{x}\right|^{2},
$$

where $c$ is the vacuum speed of light and $n_{0}$ is the nominal refractive index.

The real part of the refractive index is modified due to the Kerr effect, whose contribution to the polarization can be expressed using the linewidth enhancement factor, $\alpha: P_{\text {Kerr }}=$ $j \alpha \varepsilon_{0} \chi_{\mathrm{TPA}} E_{x}$.

The contribution of the plasma effect to the polarization is $P_{\text {plasma }}=\varepsilon_{0} \Delta \varepsilon E$, where we account for the population of carriers in all energy levels.

The simulated pulses were Gaussian shaped with a duration of $90 \mathrm{fs}$ and a pulse energy of $9 \mathrm{pJ}$. The QD amplifier was electrically driven to $13 \mathrm{kA} / \mathrm{cm}^{2}$. The QD's homogeneous linewidth and dipole moment were $1.75 \mathrm{meV}$ and $0.5 \mathrm{~nm}$, respectively. The model details and the complete set of parameters can be found in Refs. [32,35,53]. 
[1] F. Cummings, Stimulated emission of radiation in a single mode, Phys. Rev. 140, A1051 (1965).

[2] J. H. Eberly, N. B. Narozhny, and J. J. Sanchez-Mondragon, Periodic Spontaneous Collapse and Revival in a Simple Quantum Model, Phys. Rev. Lett. 44, 1323 (1980).

[3] G. Rempe, H. Walther, and N. Klein, Observation of Quantum Collapse and Revival in a One-Atom Maser, Phys. Rev. Lett. 58, 353 (1987).

[4] M. Brune, F. Schmidt-Kaler, A. Maali, J. Dreyer, E. Hagley, J. M. Raimond, and S. Haroche, Quantum Rabi Oscillation: A Direct Test of Field Quantization in a Cavity, Phys. Rev. Lett. 76, 1800 (1996).

[5] S. Haroche and J.-M. Raimond, Exploring the Quantum: Atoms, Cavities, and Photons (Oxford University Press, Oxford, UK, 2006), p. 86.

[6] D. M. Meekhof, C. Monroe, B. E. King, W. M. Itano, and D. J. Wineland, Generation of Nonclassical Motional States of a Trapped Atom, Phys. Rev. Lett. 76, 1796 (1996).

[7] M. F. Andersen, A. Kaplan, and N. Davidson, Echo Spectroscopy and Quantum Stability of Trapped Atoms, Phys. Rev. Lett. 90, 023001 (2003).

[8] M. Bellini, A. Bartoli, and T. Hänsch, Two-photon Fourier spectroscopy with femtosecond light pulses, Opt. Lett. 22, 540 (1997).

[9] V. Blanchet, M. A. Bouchène, and B. Girard, Temporal coherent control in the photoionization of cs 2: Theory and experiment, J. Chem. Phys. 108, 4862 (1998).

[10] E. O. Göbel, K. Leo, T. C. Damen, J. Shah, S. Schmitt-Rink, W. Schäfer, J. F. Müller, and K. Köhler, Quantum Beats of Excitons in Quantum Wells, Phys. Rev. Lett. 64, 1801 (1990).

[11] G. Raithel, W. D. Phillips, and S. L. Rolston, Collapse and Revivals of Wave Packets in Optical Lattices, Phys. Rev. Lett. 81, 3615 (1998).

[12] C. Wetli, S. Pal, J. Kroha, K. Kliemt, C. Krellner, O. Stockert, H. v. Löhneysen, and M. Fiebig, Time-resolved collapse and revival of the kondo state near a quantum phase transition, Nat. Phys. 14, 1103 (2018).

[13] H. Choi, V.-M. Gkortsas, L. Diehl, D. Bour, S. Corzine, J. Zhu, G. Höfler, F. Capasso, F. X. Kärtner, and T. B. Norris, Ultrafast Rabi flopping and coherent pulse propagation in a quantum cascade laser, Nat. Photon. 4, 706 (2010).

[14] I. S. Averbukh and N. Perelman, Fractional revivals: Universality in the long-term evolution of quantum wave packets beyond the correspondence principle dynamics, Phys. Lett. A 139, 449 (1989).

[15] E. L. Hahn, Spin echoes, Phys. Rev. 80, 580 (1950).

[16] H. Y. Carr and E. M. Purcell, Effects of diffusion on free precession in nuclear magnetic resonance experiments, Phys. Rev. 94, 630 (1954).

[17] M. Lorke, J. Seebeck, T. Nielsen, P. Gartner, and F. Jahnke, Excitation dependence of the homogeneous linewidths in quantum dots, Phys. Status Solidi C 3, 2393 (2006).

[18] M. Bayer and A. Forchel, Temperature dependence of the exciton homogeneous linewidth in $0.60 \mathrm{Ga} 0.40 \mathrm{As} / \mathrm{GaAs}$ selfassembled quantum dots, Phys. Rev. B 65, 041308(R) (2002).

[19] E. Waks, K. Inoue, C. Santori, D. Fattal, J. Vuckovic, G. S. Solomon, and Y. Yamamoto, Quantum cryptography with a photon turnstile, Nature (London) 420, 762 (2002).

[20] X. Li, Y. Wu, D. Steel, D. Gammon, T. Stievater, D. Katzer, D. Park, C. Piermarocchi, and L. Sham, An all-optical quan- tum gate in a semiconductor quantum dot, Science 301, 809 (2003).

[21] M. Kroutvar, Y. Ducommun, D. Heiss, M. Bichler, D. Schuh, G. Abstreiter, and J. J. Finley, Optically programmable electron spin memory using semiconductor quantum dots, Nature (London) 432, 81 (2004).

[22] I. Schwartz, D. Cogan, E. R. Schmidgall, Y. Don, L. Gantz, O. Kenneth, N. H. Lindner, and D. Gershoni, Deterministic generation of a cluster state of entangled photons, Science 354, 434 (2016).

[23] A. Faraon, I. Fushman, D. Englund, N. Stoltz, P. Petroff, and J. Vučković, Coherent generation of non-classical light on a chip via photon-induced tunnelling and blockade, Nat. Phys. 4, 859 (2008).

[24] R. Bose, D. Sridharan, H. Kim, G. S. Solomon, and E. Waks, Low-Photon-Number Optical Switching with a Single Quantum Dot Coupled to a Photonic Crystal Cavity, Phys. Rev. Lett. 108, 227402 (2012).

[25] A. Javadi, I. Söllner, M. Arcari, S. L. Hansen, L. Midolo, S. Mahmoodian, G. Kiršanskè, T. Pregnolato, E. Lee, J. Song et al., Single-photon non-linear optics with a quantum dot in a waveguide, Nat. Commun. 6, 8655 (2015).

[26] A. Y. Kitaev, Unpaired majorana fermions in quantum wires, Phys. Usp. 44, 131 (2001).

[27] L. P. Rokhinson, X. Liu, and J. K. Furdyna, The fractional ac Josephson effect in a semiconductor-superconductor nanowire as a signature of majorana particles, Nat. Phys. 8, 795 (2012).

[28] V. Mourik, K. Zuo, S. M. Frolov, S. Plissard, E. P. Bakkers, and L. P. Kouwenhoven, Signatures of majorana fermions in hybrid superconductor-semiconductor nanowire devices, Science 336, 1003 (2012).

[29] A. Das, Y. Ronen, Y. Most, Y. Oreg, M. Heiblum, and H. Shtrikman, Zero-bias peaks and splitting in an Al-InAs nanowire topological superconductor as a signature of majorana fermions, Nat. Phys. 8, 887 (2012).

[30] M. Deng, S. Vaitiekėnas, E. B. Hansen, J. Danon, M. Leijnse, K. Flensberg, J. Nygård, P. Krogstrup, and C. M. Marcus, Majorana bound state in a coupled quantum-dot hybrid-nanowire system, Science 354, 1557 (2016).

[31] S. Banyoudeh and J. P. Reithmaier, High-density $1.54 \mu \mathrm{m}$ InAs/InGaAlAs/inp (100) based quantum dots with reduced size inhomogeneity, J. Cryst. Growth 425, 299 (2015).

[32] I. Khanonkin, A. Mishra, O. Karni, V. Mikhelashvili, S. Banyoudeh, F. Schnabel, V. Sichkovskyi, J. Reithmaier, and G. Eisenstein, Ultra-fast charge carrier dynamics across the spectrum of an optical gain media based on InAs/AlGaInAs/InP quantum dots, AIP Adv. 7, 035122 (2017).

[33] L. Allen and J. H. Eberly, Optical Resonance and Two-Level Atoms, Vol. 28 (Courier Corp., North Chelmsford, UK, 1987).

[34] O. Karni, A. K. Mishra, G. Eisenstein, and J. P. Reithmaier, Nonlinear pulse propagation in inas/inp quantum dot optical amplifiers: Rabi oscillations in the presence of nonresonant nonlinearities, Phys. Rev. B 91, 115304 (2015).

[35] A. Capua, O. Karni, and G. Eisenstein, A finite-difference time-domain model for quantum-dot lasers and amplifiers in the Maxwell-Schrödinger framework, IEEE J. Select. Top. Quantum Electron. 19, 1 (2013).

[36] I. Khanonkin, A. K. Mishra, O. Karni, S. Banyoudeh, F. Schnabel, V. Sichkovskyi, V. Mikhelashvili, J. P. Reithmaier, and G. Eisenstein, Ramsey fringes in a room-temperature 
quantum-dot semiconductor optical amplifier, Phys. Rev. B 97, 241117(R) (2018).

[37] N. F. Ramsey, Experiments with separated oscillatory fields and hydrogen masers, Rev. Mod. Phys. 62, 541 (1990).

[38] T. Yajima and Y. Taira, Spatial optical parametric coupling of picosecond light pulses and transverse relaxation effect in resonant media, J. Phys. Soc. Jpn. 47, 1620 (1979).

[39] P. Borri, W. Langbein, S. Schneider, U. Woggon, R. Sellin, D. Ouyang, and D. Bimberg, Exciton relaxation and dephasing in quantum-dot amplifiers from room to cryogenic temperature, IEEE J. Select. Top. Quantum Electron. 8, 984 (2002).

[40] S. Bauer, V. Sichkovskyi, O. Eyal, T. Septon, A. Becker, I. Khanonkin, G. Eisenstein, and J. P. Reithmaier, 1.5- $\mu \mathrm{m}$ indium phosphide-based quantum dot lasers and optical amplifiers: The impact of atom-like optical gain material for optoelectronics devices, IEEE Nanotechnol. Mag. 15, 23 (2021).

[41] S. Banyoudeh, A. Abdollahinia, O. Eyal, F. Schnabel, V. Sichkovskyi, G. Eisenstein, and J. P. Reithmaier, Temperatureinsensitive high-speed directly modulated $1.55 \mu \mathrm{m}$ quantum dot lasers, IEEE Photon. Technol. Lett. 28, 2451 (2016).

[42] T. Septon, A. Becker, S. Gosh, G. Shtendel, V. Sichkovskyi, F. Schnabel, A. Sengül, M. Bjelica, B. Witzigmann, J. P. Reithmaier et al., Large linewidth reduction in semiconductor lasers based on atom-like gain material, Optica 6, 1071 (2019).

[43] J. Shah, Ultrafast Spectroscopy of Semiconductors and Semiconductor Nanostructures, Vol. 115 (Springer Science \& Business Media, New York, 2013).

[44] P. C. Becker, H. L. Fragnito, C. H. Brito Cruz, R. L. Fork, J. E. Cunningham, J. E. Henry, and C. V. Shank, Femtosecond Photon Echoes from Band-To-Band Transitions in GaAs, Phys. Rev. Lett. 61, 1647 (1988).
[45] W. Hügel, M. Heinrich, and M. Wegener, Dephasing due to carrier-carrier scattering in 2d, Phys. Status Solidi B 221, 473 (2000).

[46] S. Buchner and E. Burstein, Raman Scattering by Wave-VectorDependent Lo-Phonon-Plasmon Modes in N-InAs, Phys. Rev. Lett. 33, 908 (1974).

[47] W. M. Witzel and S. D. Das Sarma, Multiple-Pulse Coherence Enhancement of Solid State Spin Qubits, Phys. Rev. Lett. 98, 077601 (2007).

[48] O. Eyal, A. Willinger, S. Banyoudeh, F. Schanbel, V. Sichkovskyi, V. Mikhelashvili, J. P. Reithmaier, and G. Eisenstein, Static and dynamic characteristics of an inas/inp quantum-dot optical amplifier operating at high temperatures, Opt. Express 25, 27262 (2017).

[49] O. Karni, A. K. Mishra, G. Eisenstein, V. Ivanov, and J. P. Reithmaier, Coherent control in room-temperature quantum dot semiconductor optical amplifiers using shaped pulses, Optica $\mathbf{3}$, 570 (2016).

[50] R. Trebino, M. Kimmel, E. Zeek, X. Gu, P. O'Shea, A. Shreenath, S. Akturk, Q. Cao, and Z. Wang, Frequency-resolved optical gating: The state of the art, in Lasers and Electro-Optics Society, 2002. LEOS 2002. The 15th Annual Meeting of the IEEE, Vol. 1 (IEEE, New York, 2002), pp. 223-224.

[51] A. Icsevgi and W. Lamb Jr, Propagation of light pulses in a laser amplifier, Phys. Rev. 185, 517 (1969).

[52] N. Suzuki, FDTD analysis of two-photon absorption and free-carrier absorption in Si high-index-contrast waveguides, J. Lightwave Technol. 25, 2495 (2007).

[53] A. K. Mishra, O. Karni, and G. Eisenstein, Coherent control in quantum dot gain media using shaped pulses: A numerical study, Opt. Express 23, 29940 (2015). 